Research Article

\title{
Characterization and Synthesis of Selenium -TPGS Nanoparticles for Target Delivery Clove to Minimize Cytogenic and Liver Damage Induced in Adult Male Rats
}

\author{
Adnan Mansour Jasim ${ }^{1}$, Essa Daham Alhtheal $^{2}$, Saad Saleem Raheem ${ }^{3}$, Kareem Judi Rawaa ${ }^{4}$, \\ Abbas Hamad ${ }^{5}$ \\ ${ }^{1}$ College of Veterinary Medicine, Al-Qasim Green University Iraq. \\ ${ }^{2}$ University of Technology / Nanotechnology and Advanced Research Center, Iraq. \\ ${ }^{3}$ University of Al-Furat Al-Awsat Technology, College of Healthy and Medical Techniques, Iraq. \\ ${ }^{4}$ Babylon Veterinary Teaching Hospital. \\ ${ }^{5}$ College of Biotechnology, Al-Qasim Green University Iraq.
}

—Corresponding authors. E-mail: adnan.mansouri81@vet.uoqasim.edu.iq; dr.abbashamad@biotech.uoqasim.edu.iq

Received: Dec. 5, 2020; Accepted: Jan. 24, 2021; Published: Apr. 16, 2021

Citation: Adnan Mansour Jasim, Essa DahamAlhtheal, Saad Saleem Raheem, Kareem Judi Rawaa, and Abbas Hamad, Characterization and Synthesis of Selenium -TPGS Nanoparticles for Target Delivery Clove to Minimize Cytogenic and Liver Damage Induced in Adult Male Rats. Nano Biomed. Eng., 2021, I3(2): I27-I36.

DOI: 10.5101/nbe.v13i2.p127-136.

\begin{abstract}
Nanoparticle science is currently an area of intense scientific research due to a wide range of possible applications in the biomedical and electronic fields. Nanoparticle works as an important bridge between bulk materials and atomic or molecular structures. The aim of this study is to evaluate the preparation of clove buds by extraction and encapsulation using nanoprecipitation technique with selenium nanoparticles. The nanoparticles are prepared by nanoprecipitation technique and characterized by particles sizers, UV spectrophotometers, and scanning electron microscopy (SEM) images which were utilized for regular distribution and spherical shape of nanoparticles with the size range of $206.5 \mathrm{~nm}$; active compounds were detected by Gas chromatography-mass spectrometry (GC-MS). The antioxidant activity of clove nanoparticles was evaluated against hepatotoxic thioacetamide in male rats. 30 albino male rats were divided into 6 groups, with 5 rats in each group. All groups of animals were treated with single dose of Thioacetamide100 mg/kg except the control. Groups T2 and T3 received Thioacetamide (100)/kg) I.p., treated orally with $100 \mathrm{mg} / \mathrm{kg}$ of clove extract and clove nanoparticles of dose by day respectively, while the last group was treated with selenium nanoparticles at dose $0.5 \mathrm{mg} / \mathrm{kg}$ daily. The selenium loaded clove nanoparticles showed spherical shape with seller size of $206.5 \mathrm{~nm}$. GCMS of Syzygium aromaticum (clove) contained more than 13 active compounds. Consequently, the cytogenic study of clove nanoparticles showed a clear reducing of micronuclei percentages, chromosomal aberration and their types. In addition, the serum evaluated in this study showed significant reduction of TNFa ng/dl and IL6 in rats treated with clove extract and clove-nanoparticles in comparison with thioacetamide alone. Interestingly, the antioxidant activity of GPX was significantly elevated in rats having received clove-nanoparticles. Additionally, the histopathological defect such as inflammation and necrosis in liver and kidney was cured by using clove nanoparticles. The clove loaded selenium nanoparticles illustrated strong antioxidant and scavenging activities led by alleviate cytogenic and tissue damage induced by thioacetamide.
\end{abstract}

Keywords: Thioacetamide, Clove nanoparticles, Selenium, Antioxidant, GSMS 


\section{Introduction}

The use of herbal medicines and supplements has increased dramatically over the past three decades, with no less than 80 percent of individuals worldwide relying on them for some type of primary health car [1]. The fact that closely related plants have the same or identical chemical profiles is the basis of chemotaxonomy [2, 3]; therefore, in different diseases, plants are used as complementary and alternative medicine.

Syzygium aromaticum (clove) has many pharmacological activities and is a popular spice used for food preservation, in particular, in meat production, chemical preservatives are substituted because of their antioxidant and antimicrobial properties [4]. However, among other spices, the clove has gained considerable interest because of its high antimicrobial and antioxidant activities [5]. Clove flower buds have more than 18 percent of volatile oil containing $\beta$-cariofilenoeugenol acetate and eugenol, which confirmed vitro antioxidant effectiveness since it enables the hydrogen atom to be donated and therefore fixes the radical phenoxil, which results in the formation of steady molecules that do not produce or increase oxidation [6]. Ethanolic extract of S. Aromaticum, in addition, exhibited powerful antioxidant activity that results in hepatoprotective activity against paracetamol causing liver damage due to inhibition of intracellular enzymes via efficacy of membrane stabilization with tissue regeneration repair mechanism [6].

Han and parker [7] reported that S. aromaticum had antiviral, antifungal, antimicrobial, antioxidant, anticancer, and anti-inflammatory properties, due to the active constituent eugenol. Curiously, it has been shown that $\mathrm{S}$. aromaticum inhibits cytotoxic activities and inhibits human tumor cell lines PC-3 and Hep G2, which cause dehydrodieugenol-related death of human cancer cells [8]. In addition, Clove oil and eugenol play a key role in the fight against breast, leukemia, lung, and colorectal tumor cells by anticancer activities. Clove oil has an immunomodulatory and anti-inflammatory effect through the suppression of the pathway of nuclear factor $-\kappa \mathrm{B}(\mathrm{NF}-\kappa \mathrm{B})$ and lipopolysaccharide (LPS) action [8, 9]. In HL60 cancer cells, Eugenol displayed various degrees of cytotoxicity and inhibited cell growth by 50 percent at a concentration of $23.7 \mu \mathrm{M}$. The findings showed a substantial increase in eugenol-induced fragmented
DNA. Eugenol also has the potential to induce ROSdependent apoptosis induction in a line of HL60 cells [8]. On the other hands, eugenol and some alkaloids such as sanguinarine and benzo have been found to defend the hepatocyte against hepatotoxicity in male rats induced by CCl4 [10]. In conventional biological imaging of cells and tissues, nanoparticles can provide essential improvements. There are various benefits of nanoparticles to modern medicine [11]; the concentration of drugs in target tissues has been improved; hence, lower doses of the drug are required. Furthermore, decreasing patient-to-patient variability, increasing solubility, increasing oral bioavailability, increasing dissolution rate, increased surface area, a limited amount of dosage demand and a quicker initiation of therapeutic action are some of the benefits of nanosizing [12]. Moreover, some specific characteristics that can be promoted to expand distribution, improve drug localization and improve drug efficacy have been achieved by drugs that are conveyed to the nano range [13]. In our research, improving herbal medicine through nanotechnology enhances the pharmacological activity of traditional therapeutic medicinal plants, phytometabolites and metabolomics, and the pharmacological activity of these nanoparticles in the field of delving deeper.

\section{Experimental}

Martials vitamin E-TPGS was purchased from MCE, USA, Eliza kit Rat glutathione (GSH) SL0998Ra. This study was conducted at the period January - March, 2020 in pharmacology department of veterinary medicine of AL-Qassim Green University.

\section{Preparation of clove extracts}

Clove bud was dried in oven $\left(40{ }^{\circ} \mathrm{C}\right)$ and ground to fine powder in a mill. Milled material (200 g) was extracted individually with organic solvents with 200 $\mathrm{mL}$ ethanol $70 \%$ and distilled water using magnetic stirrer with temperature $40{ }^{\circ} \mathrm{C}$ and by filtration by filter paper (Whatman No. 1). The period of extract was $4 \mathrm{~h}$. The remaining were re-extracted under the same manner. The extract was collected after solvent evaporated by oven under $45{ }^{\circ} \mathrm{C}$. The extract was weighed to determine the dried yield then stored at 20 ${ }^{\circ} \mathrm{C}$ for further analyses [12].

\section{Preparation of clove-SE-TPGS nanoparticles}

Nanoparticles of clove extracts were prepared by the nanoprecipitation method according to [14] with 
mild modification. The organic solution including 100 $\mathrm{mg}$ of clove was placed in a glass test tube, and then transferred $1 \mathrm{~mL}$ of acetone solvent, a small piece of aluminum foil, and Parafilm the foil used to close the tube then incubated overnight; then vortex on high speed until all extract was entity dissolved ( 10 min). An aqueous phase was prepared by adding $100 \mathrm{~mL}$ of $0.03 \% \mathrm{w} / \mathrm{v}$ Vitamin E-TPGS and $100 \mathrm{mg}$ of selenium nanoparticles to a $200 \mathrm{~mL}$ glass beaker for overnight. After that, put on a magnetic stir bar at the stirring speed $500 \mathrm{rpm}$. Then, the test tube was stirred. $100 \mu \mathrm{L}$ of clove extract was immediately added to the aqueous phase of TPGS-selenium under high stirring by vortex at $800 \mathrm{rpm}$ for $5 \mathrm{~min}$, until all quantity of extract added to TPGS. The emulsified clove extract solution was put in the small beaker $20 \mathrm{~mL}$, and then directly transferred to the ultrasonicator and immersed in the ice water and sonicate the emulsion for $9 \mathrm{~min}$, pulse on time 15 seconds with pulse off time 15 seconds and 50\% amplitude. The emulsified nanoparticle was put in beaker $100 \mathrm{~mL}$ and then wrapped in aluminum foil, the top of beaker was leaved open to facilitate solvent evaporation. The suspension obtained was filtered (Whatman filter paper 1) to discard any precipitated and then centrifuged at $14000 \mathrm{rpm}$ at 4 ${ }^{\circ} \mathrm{C}$. The supernatant containing the unbound drug was discarded; the pellet obtained was washed 2-3 times with distilled water.

\section{Characterization of clove-SE nanoparticles coated by TPGS}

This step can be conducted using apparatus particle size, UV-visible spectrometry and scanning electron microscope (SEM). For this purpose, nano zeta sizer (Malvern Instruments, UK) was used.

\section{Experimental study}

30 healthy male rats weighing 200-250 g and aged 3 months were used in this study; they were obtained from the animal house colony in veterinary medicine and kept for 14 days as acclimatization period before the beginning of the experiment. The animals were maintained at standard housing conditions and fed with a standard pellet diet, and water was given ad libitum.

\section{Induction of liver damage in male rats}

30 albino male rats were colonized in animal house of AL-Qasim Green University of Veterinary Medicine. The rats were randomly divided into 5 equal groups. Group I: Control: Animals in this group received normal saline as negative control.
Group II: T1: Animals in this group were offered oxidative stress by thioacetamide at $100 \mathrm{mg} / \mathrm{kg}$. Group III: T2: Animals in this group were given a single dose injection by thioacetamide at $100 \mathrm{mg} / \mathrm{kg}$ I.p. and treated orally with $100 \mathrm{mg} / \mathrm{kg}$ daily of clove extract. Group IV: T3: Received thioacetamide at 100 $\mathrm{mg} / \mathrm{kg}$ I.p. and treated orally with $100 \mathrm{mg} / \mathrm{kg}$ daily of clove nanoparticles. Group VI: T4: Rats received thioacetamide at $100 \mathrm{mg} / \mathrm{kg}$ I.p. and treated orally with selenium nanoparticles at the concentration of $0.5 \mathrm{mg} /$ $\mathrm{kg}$ [15]. The experiment was carried out for 8 weeks.

\section{Statistical analysis}

The statistical analysis was carried by using complete randomized design (CRD) method according to AL-Rawi and Kalaf-Allah [33]. The mean differences between the averages of the studied traits were determined at the probability level of 0.01 using the Duncan test [38].

\section{Elaboration of karyotype of bone marrow cells}

The chromosomes were prepared via direct method (Sharma et al. [16]) with some modifications.

\section{Micronucleus formation assay}

To perform the appreciation of micronucleus formation, the procedure of [17] was apply.

\section{Results and Discussion Chemical composition of Syzygium aromaticum extract}

Table 1 shows that 13 essential compounds were detected in CEO by GC/MS. The main compounds were eugenol and $\beta$-caryophyllene that are in concord with previous study published in literature [34, 35].

Table 1 Chemical components of extracted clove determined by GC/MS

\begin{tabular}{cc}
\hline Component & Retention time \\
\hline Humulene & 1 \\
Gallic acid & 3 \\
Stigmasterol & 18 \\
Cosuarinine & 37 \\
Querecetin & 53 \\
Caryophyllene alcohol & 1593 \\
Caryophyllene & 1583 \\
Eugenyl acetate & 1522 \\
Trans-isoeugenol & 1449 \\
Caryophyllene $\beta$ & 1415 \\
Eugenol & 1359 \\
Methyl salicylate & 1190 \\
Limonene oxide & 1029 \\
\hline
\end{tabular}


The high level of antioxidant activity in clove may arise from its hydrogen donating capacity inhibited by a broad range of ingredients like eugenol, eugenyl acetate, phenylpropanoids, dehydrodieugenol, transconiferyl aldehyde, $\beta$-caryophyllene, 2-heptanone biflorin, kaempferol, rhamnocitrin, myricetin, ellagic acid and oleanolic acid [34, 36]. Moreover, clove contains quercetin, kaempferol stigmasterol and gallic acid has antiulcer, antinocieptive, anti-proliferative, and anti-inflammatory properties [37].

\section{Characterization of clove-selenium nano- particles}

The results regarding particle size and polydispersity index (PDI) of clove-loaded selenium NPs coated with TPGS are presented in Figs. 1-3; they were found to have a mean diameter of $206.5 \mathrm{~nm}$ and $199.9 \pm$ $14.7 \mathrm{~nm}$, respectively as well as rounded shape of nanoparticles was seen in Fig. 2 and 3 and Table 1. The size of the NPs was increased by an increment of the clove/selenium ratio, which was in accordance with the results of $[18,19]$. PDI was employed to determine the distribution of the particle size in suspensions. The results showed a lower PDI at 0.005 , which indicates a more homogenous particle size distribution and a stable particle population of uniform distribution (Hasani et al., 2015). It is a fact that natural products are a healthier match than synthetic drugs. Hence, a more global collection of extract from plants can supply many novel chemicals in drug detection screening for health problems.

\section{Micronuclei deformity}

In the present study rats administrated with thioacetamide showed significant $(\mathrm{p}<0.05)$ increase in aberrant metaphases, when compared to the control group and others treated groups (Fig. 4-7). The toxic effect of TAA due to metabolizing by liver CYP 450 2E1 enzymes to active metabolites include sulfone, sulfoxide, acetamide, sulphate that were responsible for genotoxic, oxidative stress and hepatotoxic [20]. Moreover, thioacetamide-5-oxide is accountable

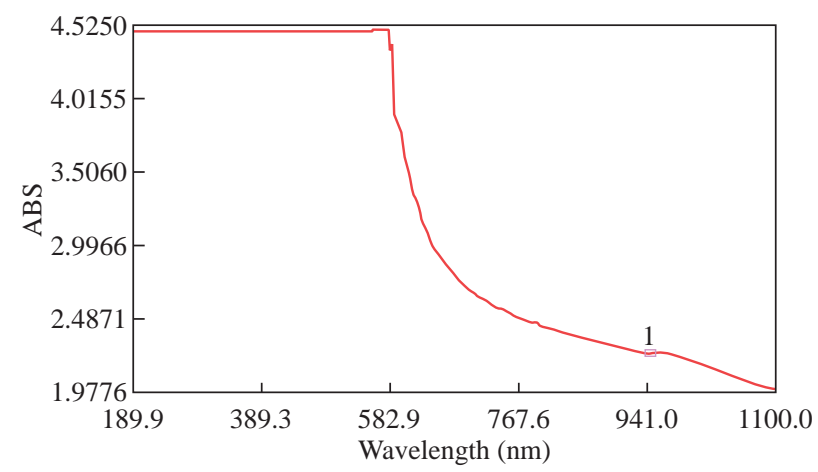

Fig. 1 UV-visible spectrum of clove NPs; lambda was 946.90 $\mathrm{nm}$ and absorbance value was 2.2469 .

15-0 (Combined)

Effective Diameter: $206.5 \mathrm{~nm}$

Polydispersity: 0.005

Baseline Index: 8.5/100.00\%

Elapsed Time: 00:01:30

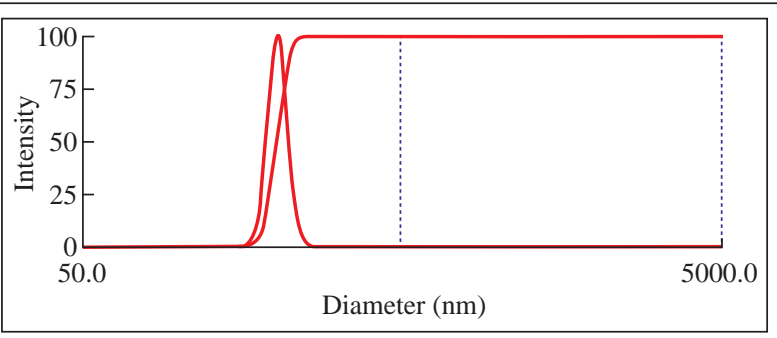

Lognormal Distribution

Fig. 2 Histogram of clove NPs effective diameters $=206.5 \mathrm{~nm}$.
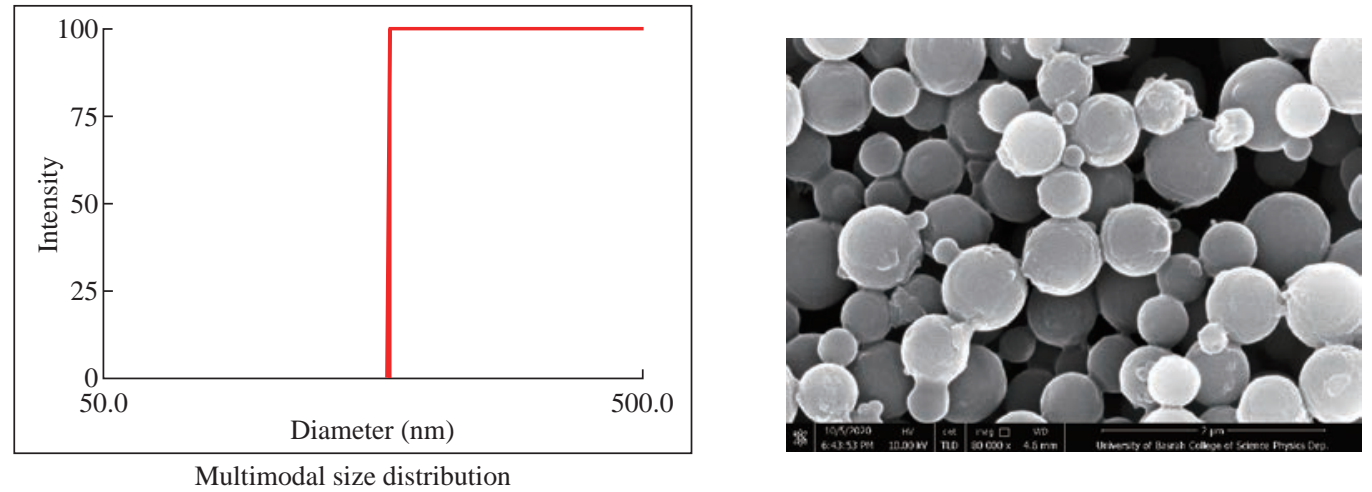

Fig. 3 Histogram of clove NPs with mean of particle size was $199.9 \pm 14.7 \mathrm{~nm}$ and SEM illustrating the morphology. 

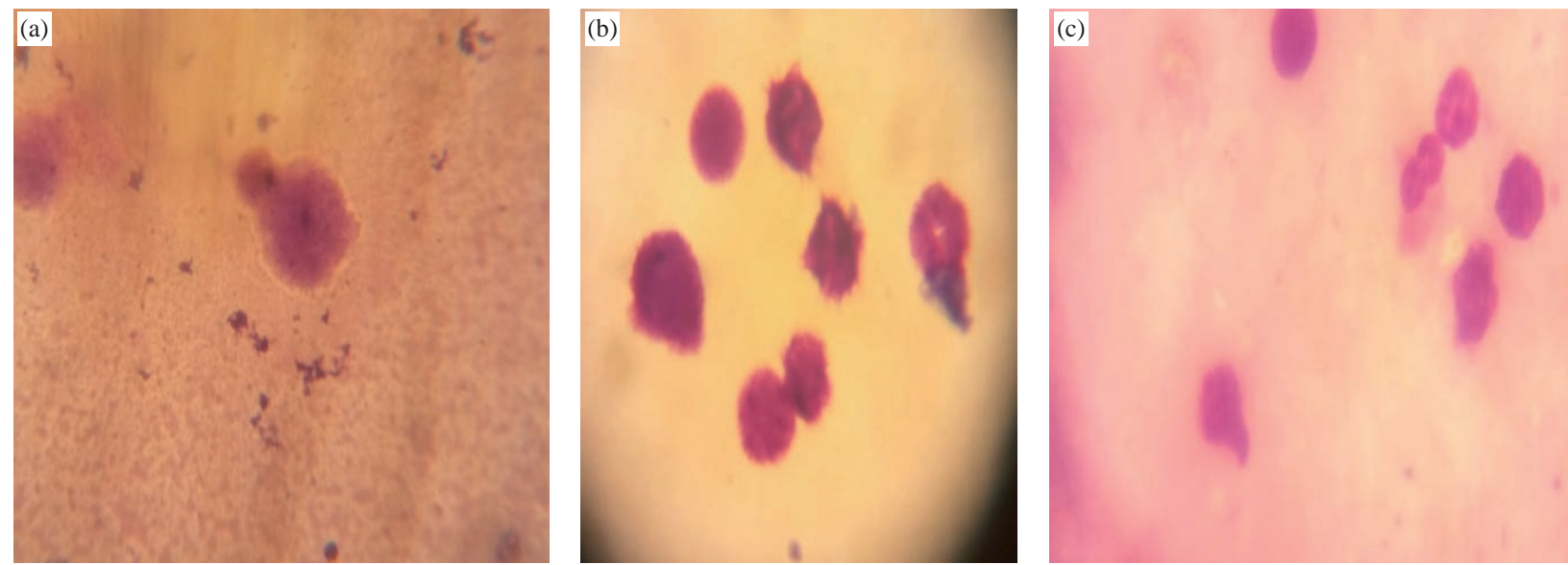

Fig. 4 (a) and (b) Micronuclei deformity from bone marrow cell rats received thioacetamide; (c) Bone marrow cell rats received thioacetamide and treated with extract clove extract.
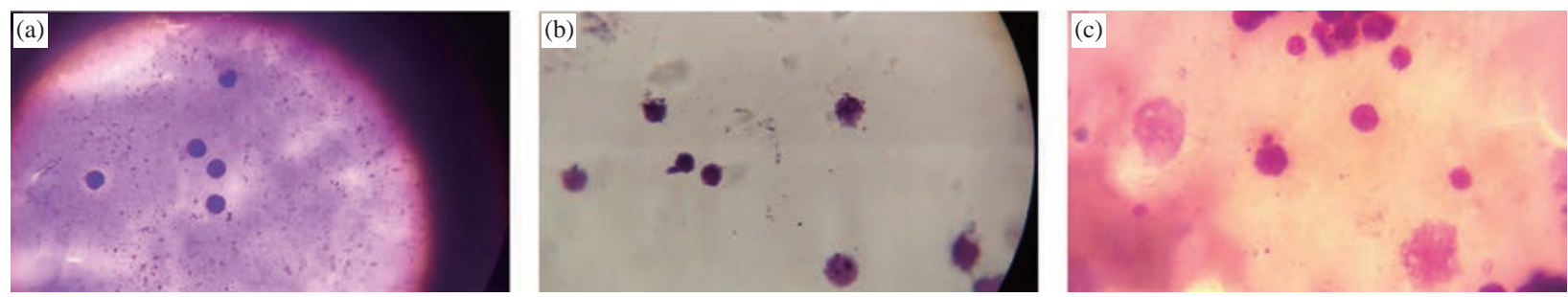

Fig. 5 (a) Micronuclei from bone marrow cell rats negative control group; (e) and (f) Bone marrow cell rats received thioacetamide and treated selenium nanoparticles and clove-nanoparticles respectively.

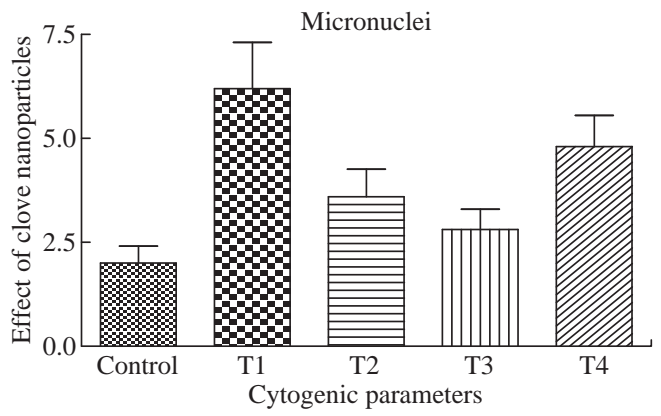

Fig. 6 Effect of clove nanoparticles in micronuclei of bone marrow cell in rats having received thioacetamide. T1, T2, T3, T4 and T5 illustrated the normal control, thioacetamide and clove extract treated, clove-nanoparticles and selenium nanoparticles treated groups respectively.

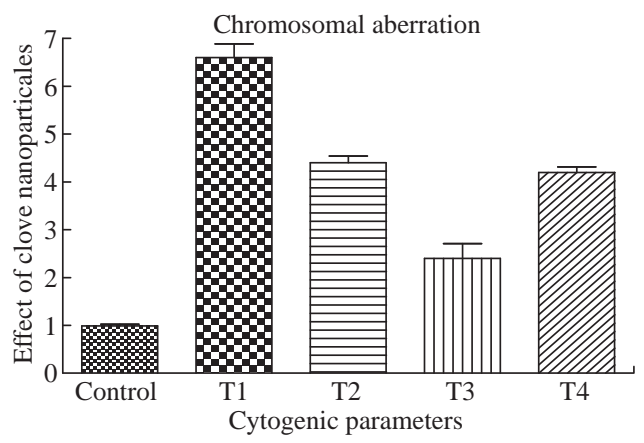

Fig. 7 Effect of clove nanoparticles in chromosomal aberration of bone marrow cell in rats having received thioacetamide. T1, T2, T3, T4 and T5 illustrated the normal control, thioacetamide and clove extract treated, clove-nanoparticles and selenium nanoparticles treated groups respectively. for the alteration in cell permeability, inhibits mitochondrial activity and raises the intracellular concentration of calcium which leads to cell death [21] (Fig. 8 and 9). TAC is oxidative stress on the antioxidant system, liver tissue and nucleic acids, as well as their mutagenic activity, which was improved and readjusted via administration of alcoholic extracts of clove and their selenium-clove nanoparticles. On the other hand, no data was recorded in literature about these synthesis clove nanoparticles and their effect to reduce mutagenic activity of TAC in albino rats. The present findings of percent micronuclei was significantly reduced $(p \geq 0.05)$ in rats having received nanoparticles to recorded mean value of $2.81 \pm 0.84$

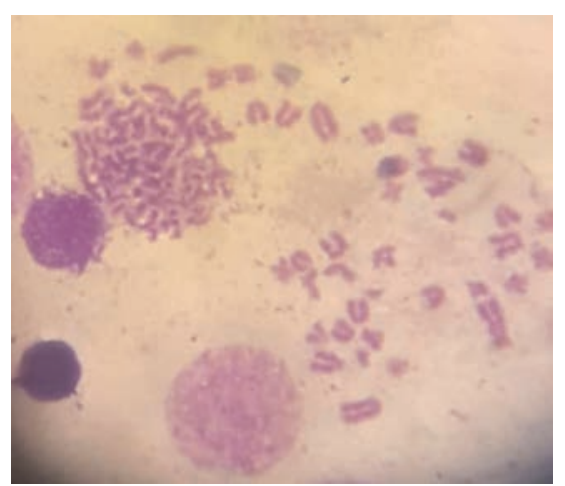

Fig. 8 Metaphase chromosomes of bone marrow cells showing fragment chromosome and dicentric chromosome $\times 100$. 


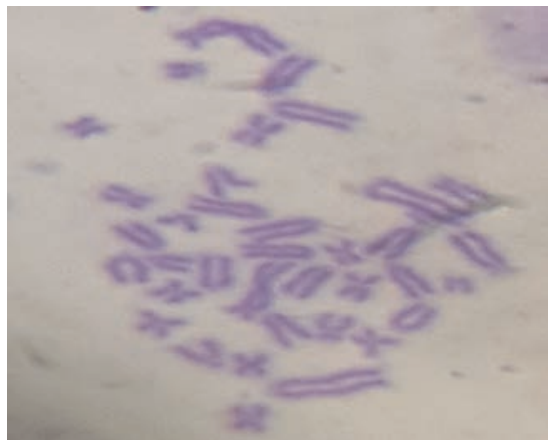

Fig. 9 Metaphase chromosomes of bone marrow cells showing chromosome breakage and long chromosome.

Table 2 Particle size, zeta potential, polydispersity index (PDI), and retention value of CEO-loaded chitosan nanoparticles

Organic phase Aqueous phase Particle size (nm) Polydispertion index

\begin{tabular}{cccc}
\hline Acetone & TPGS & 206.5 & 0.005 \\
\hline DMSO & PVA & 295.6 & 0.315 \\
Hexane & Tween 80 & 390 & 0.266 \\
\hline
\end{tabular}

as compared to $3.60 \pm 1.14$ with the extract of clove only; thus, the results of chromosomal aberrations were deceased in percentage and their types in cloveselenium nanoparticles among other treated groups (Table 2). Encapsulation process or attachment of clove extract improves components with selenium, but also it enhances the antioxidant activity of these bioactive compared to their free clove as a result of protection against the adverse effects of oxidative stress (Table 3). Hosseini et al. [19] confirmed that clove oil loaded with chitosan has antioxidant activity higher than nonencapsulated CEO.

\section{Effect of clove nanoparticles on antioxidant and cytokines}

Current study displayed that clove-selenium nanoparticles has a significant $(\mathrm{p} \leq 0.05)$ raise in serum glutathione rats that uncover to thioacetamide oxidative stress when compared with reference clove extract (Fig. 12). Clove is a rich source of bioactive compounds especially phenolic compound that has antioxidant activity to remove [22]. In addition, clove oil had an effective DPPH scavenging it has antioxidant activities similar to a-tocopherol and trolox as related antioxidant compounds so that potent inhibitor for lipid peroxidation of linoleic [23]. In oxidative stress, GSH acts to neutralize the effect of generated peroxides. Our data suggest the clove nanoparticles and their extract might exert its activity via ameliorating oxidative stress and inflammation. Clove extract contain A large number of active compound, have been responsible from many pharmacological activity, such compound the phenylpropanoids, alkaloids, amino acids, flavonoids, $\beta$-carotene, $\beta$-caryophyllene, $\beta$-pinene, $\beta$-sitosterolglucoside, proteins, sterols, reducing sugars, tannins, phenols, ascorbic-acid, benzyl-acetate carvone, furfural, stigmasterol, aluminum, zinc, boron, calcium, manganese kaempferol, mucilage, naphthalene, and oleanolic acid [24]. Glutathione peroxidase (GPx) is a selenoprotein with biological properties that permit the detoxification of ROS. GPx has an essential role to prevent oxidative damage of cellular constituents via controls the status of a redox system. Moreover. GPX is the first line of defense against free radicals. Its deficiency leads to dyslipidemia, insulin resistance, inflammation, and metabolic alterations, which leads to the progress of cardiovascular and degenerative diseases as well as promotes the oxidation of proteins and deoxyribonucleic acid (DNA) that encourage to carcinogenesis and mutagenesis [25].

Significant increase in the serum TNF- $\alpha$ and IL6 when TAC was administrated, compared to the negative control group and the group treated with clove nanoparticle extract and selenium nanoparticles (Fig. 10 and 11). This finding is in agreement with those of Chen et al. [25] who confirmed that a single dose of TAC could cause a significant raise of TNF- $\alpha$ and IL6 serums in rats, reporting that the mice treated with clove extract showed inhibition of macrophages to produce both IL-1B and IL-6. The essential oil of clove at different concentrations could inhibit

Table 3 Effect of clove nanoparticles in cytogenic parameters chromosomal aberration (\%) in male rat bone marrow

\begin{tabular}{cccccc}
\hline Chromosome & C & T1 & T2 & T3 & T4 \\
\hline Ch. breakage (\%) & $0.33 \pm 0.04 \mathrm{~B}$ & $1.54 \pm 0.10 \mathrm{~A}$ & $0.95 \pm 0.05 \mathrm{~A}$ & $0.70 \pm 0.16 \mathrm{~B}$ & $0.65 \pm 0.19 \mathrm{~B}$ \\
Ch. fragment (\%) & $055 \pm 0.02 \mathrm{C}$ & $1.52 \pm 0.08 \mathrm{~A}$ & $0.94 \pm 0.098 \mathrm{~B}$ & $0.60 \pm 0.19 \mathrm{C}$ & $0.94 \pm 0.03 \mathrm{~B}$ \\
Ch. ring (\%) & $0.00 \pm 0 \mathrm{C}$ & $1.40 \pm 0.01 \mathrm{~A}$ & $0.88 \pm 0.09 \mathrm{~B}$ & $0.70 \pm 0.018 \mathrm{~B}$ & $0.87 \pm 0.011 \mathrm{~B}$ \\
Ch. long (\%) & $0.00 \pm 0.00 \mathrm{C}$ & $0.94 \pm 0.13 \mathrm{~A}$ & $0.52 \pm 0.14 \mathrm{~B}$ & $0 \pm 0.00 \mathrm{~B} \mathrm{C}$ & $0.64 \pm 0.20 \mathrm{~B}$ \\
Hypo ploidy (\%) & $0.00 \mathrm{C}$ & $1.22 \pm 0.13 \mathrm{~A}$ & $0.96 \pm 0.17 \mathrm{~B}$ & $0 \pm 0.00 \mathrm{C}$ & $0.80 \pm 0.15 \mathrm{~B}$ \\
\hline
\end{tabular}

Note: Different capital letters indicate significance $(\mathrm{P} \leq 0.05)$ among groups. T1, T2, T3, T4 and T5 illustrate normal control, thioacetamide and clove extract treated, clove-nanoparticles and selenium nanoparticles treated groups respectively. 


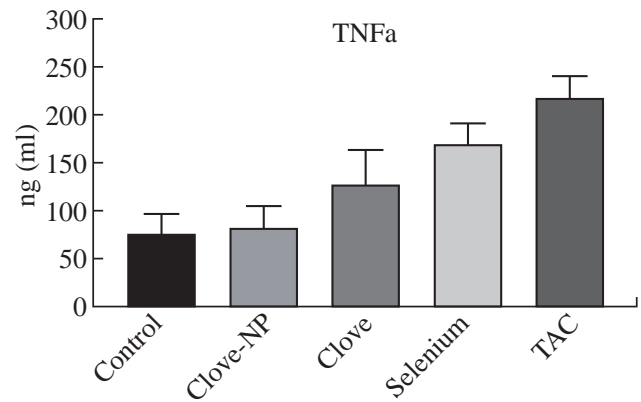

Fig. 10 The serum level of TNFa ng/dL showed a significant reduction in clove nanoparticles $(p \leq 0.05)$ especially in rats having received clove extract and clove-nanoparticles.

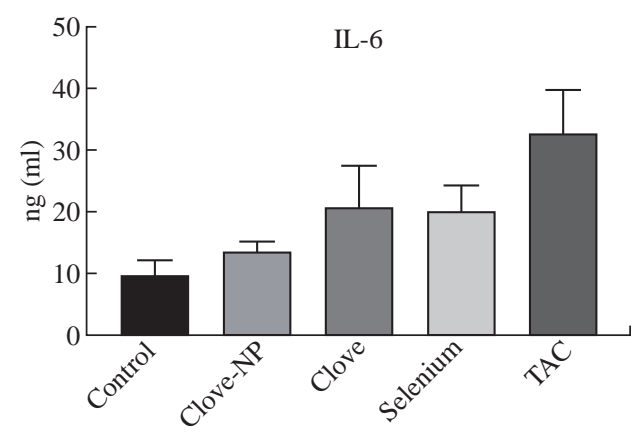

Fig. 11 The serum level of IL6 ng/dL showed a significant reduction in clove nanoparticles $(p \leq 0.05)$ especially in rats having received clove extract and clove-nanoparticles.

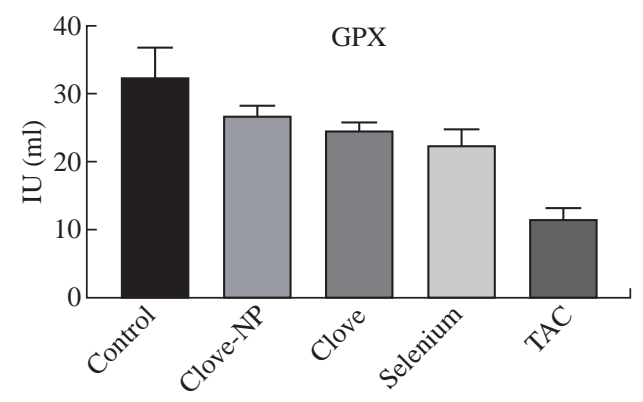

Fig. 12 The serum level of GPX iu/mL showed a significant elevation in clove nanoparticles $(p \geq 0.05)$ especially in rats having received clove extract and clove-nanoparticles and selenium nanoparticles.

the production of these cytokines in vitro. Eugenol, a major component of the clove extract, maybe a causative agent of cytokine inhibition. Many studies reported that clove's active compound, eugenol and isoeugenol, have antigenotoxic, antioxidant, antiinflammatory and anticarcinogenic activities. In addition, they have been shown to modulate several immune responses, including an anti-inflammatory effect, but the mechanism underlying their effects has not been fully explained [25, 28].

\section{Histopathological study}

Histologically (Fig. 13-20), TAA administration leads to evident alteration in the liver tissue architecture and produces inflammation and necrosis a significant promotion in serum levels of AST, ALT, ALP, bilirubin, total cholesterol, triglyceride and LDL with a reduction in the levels of total proteins, albumin and HDL [25]. In our study, TAA administration produced focal aggregation of inflammatory cells with vacuolation in the liver parenchyma and hepatocyte necrosis. Our histological results showed that rat livers administered with thioacetamide and treated with clove extract showed dilatation of sinusoid, with focal MNCS cell aggregation and slight vacuolation in the liver parenchyma. Gashlan and Al-Beladi [29] reported that diabetic rats treated with clove oil revealed an

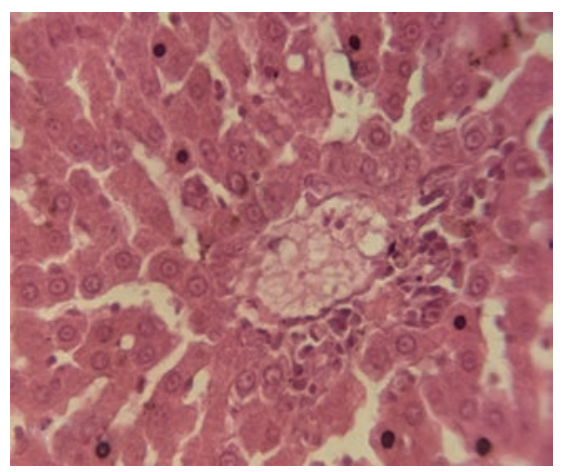

Fig. 13 Histopathological section in the liver of group T2 administrated with thioactamide and treated with clove extract showed dilatation of sinusoid, with focal MNCS cell aggregation slight vacuolation in the liver parenchyma.

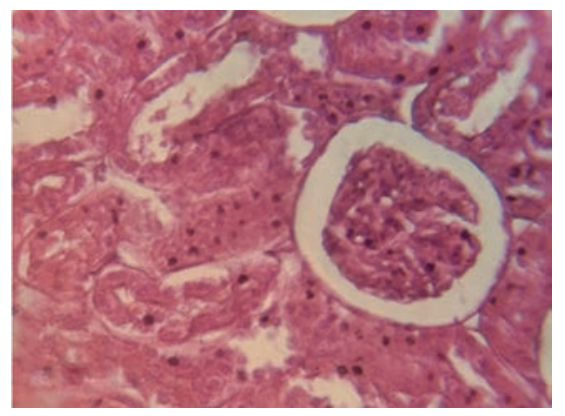

Fig. 14 Histopathological section in the kidney of group T2 administrated with thioacetamide and treated with clove extract showed dilated tubules with slight shrinkage of glomerular tuft.

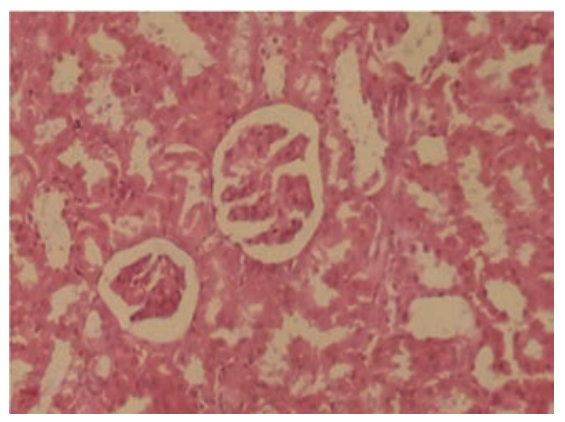

Fig. 15 Histopathological section in the thioacetamide without treatment for 8 weeks showed degenerated and shrunk glomerular tuft (H\&E). 


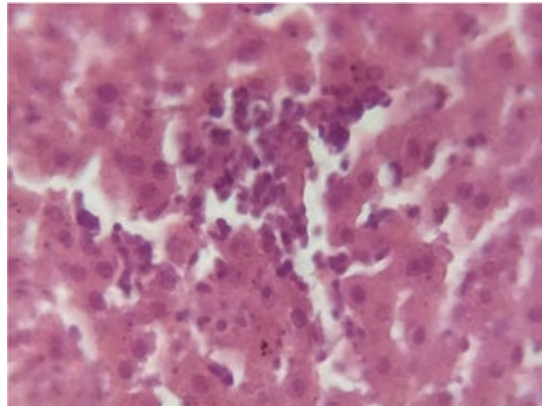

Fig. 16 Histopathological section in the liver of group T1 administratd with thioacetamide and without treatment for 8 weeks showed focal aggregation of inflamatory cells with vacuolation in the liver parenchyma and necrosis of hepatocyte.

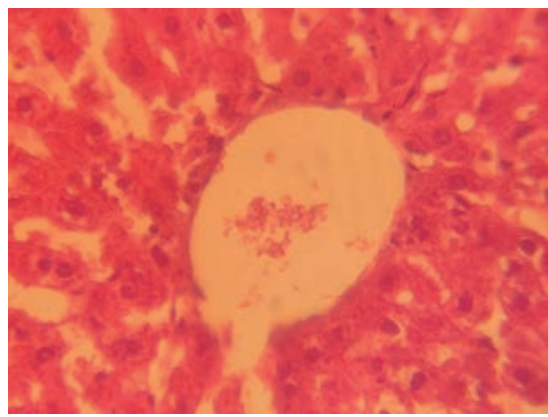

Fig. 17 Histopathological section in the liver of group T4 administratd with thioacetamide and treated for 8 weeks with clove nanopartices showed slight vacuolation in the liver parenchyma.

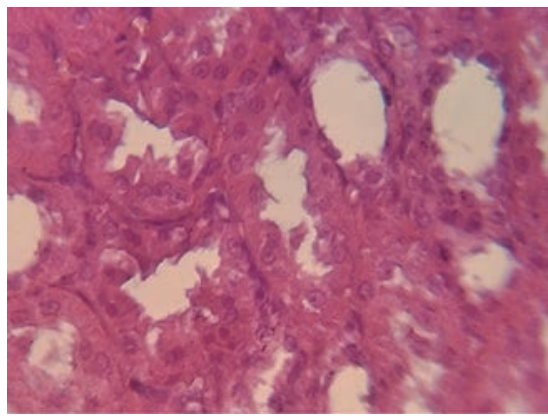

Fig. 18 Histopathological section in the kidney of group T4 administratd with thioacetamide and treated for 8 weeks with clove nanopartices showed slight atrophic tubules with infiltration and dilation of the lumen of renal tubules.

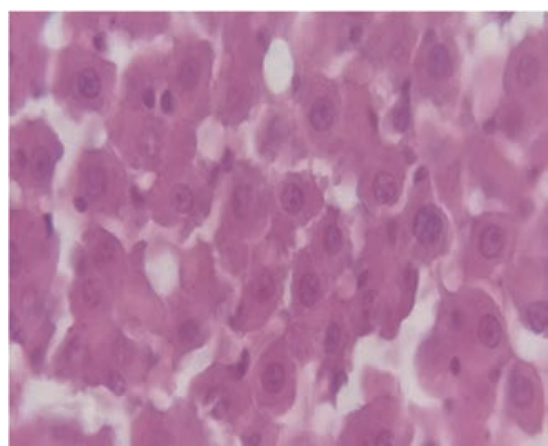

Fig. 19 Histopathological section in the liver of group T5 administrated with thioaetamide and treated selenium nanoparticles showed slight vacuolation in the liver parenchyma with dilatation of sinusoid and activation of kupffer cells.

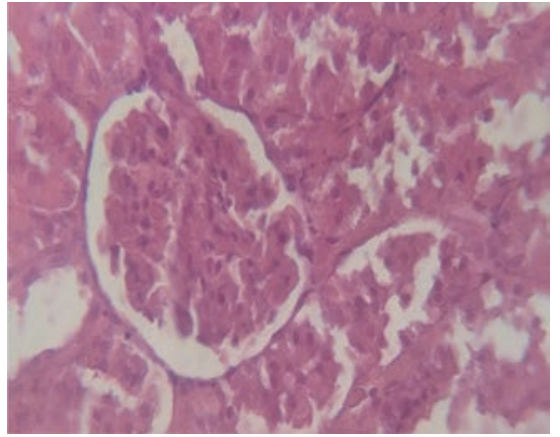

Fig. 20 Histopathological section in the kidney of group T5 administrated with thioaetamide and treated selenium nanoparticles showed hyaline cast with sloughed brush border.

elevation in antioxidant enzyme activities compared to the untreated diabetic animal, and they further noted that clove oil treatment tends to help the cells become normal. Eugenol active constitutes of clove return the liver function to near-normal by repressing lipid peroxidation and the release of cytokines recorded via reducing IL6 and raising SOD and GPx that minimise generation of ROS and reduction in inflammatory cell infiltration [33]. One possible mechanism is that clove nanoparticles act synergistically with selenium to support the immune system and raise the antioxidant enzyme that controls complications from toxic compounds.

Histopathological section of kidney of rats administrated by thioacetamide alone showed degenerated and shrinkage glomerular tuft. On the other hand animal that received treatment by selenium nanoparticles, clove extract and clove nanoparticles loaded with selenium showed clear improvement of renal tissue by reduce its damage. The present study showed that kidney rats administrated by thioacetamide and treated for eight weeks with Clove nanoparticles show slight atrophic tubules with infiltration and dilation of the lumen of renal tubules. Moreover that clear improvement may be due to synergistic action of both clove constitute and selenium that lead to enhancement antioxidant activity and increase of glomerular filtration to remove toxic compound related with thioacetamide. The recent study was harmony with Nasri et al. [30] that illustrated clove buds (S. aromaticum) can beneficially ameliorate kidney damage induced by iodixanol. Abozid and coworker indicate that clove extract has hepatoprotective and reno protective effects versus oxidative stress result from hydrogen peroxide. The new study reported that essential oil clove significantly diminish the biochemical and histological changes of kidney tissue 
induced by lead-intoxication in rats [31]. To find the effectiveness of clove extract against methotrexate induced hepatotoxicity and nephrotoxicity in rats, it was concluded that rats administrated by methotrexate showed clear degeneration, inflammatory cell infiltration, necrosis, and hemorrhage in renal tissues while these changes significantly reduced after rats received clove extraction [32].

\section{Conclusions}

Clove extract has low solubility in water; hence, encapsulation techniques were applied to increase their dispensability in aqueous media and stability by using TPGS stabilizer as well as encapsulation, which brought about a good compatibility between the clove and selenium. The clove loaded selenium NPs illustrated strong antioxidant and scavenging activities resulted in cytogenic reduction in adult male rats.

\section{Acknowledgements}

The authors would like to thank to the Department of Nanotechnology, the Rector of Universities of Technology, the dean of the Veterinary Medicine, assistant prof. Dr Mohammed Jawad, and Institute of Science and Technology in Medicine Keele University for supporting us in the experiment.

\section{Conflict of Interests}

The authors declare that no competing interest exists.

\section{References}

[1] M. Ekor, The growing use of herbal medicines: issues relating to adverse reactions and challenges in monitoring safety. Frontiers in Pharmacology, 2014, 4: 177.

[2] R. Batool, M.R. Khan, M.A. Sajid, et al., Estimation of phytochemical constituents and in vitro antioxidant potencies of Brachychiton populneus (Schott \& Endl.). $R$. Br. BMC Chemistry, 2019, 13(1): 32.

[3] D. Hao. P. Xiao, Pharmaceutical resource discovery from traditional medicinal plants: Pharmacophylogeny and pharmacophylogenomics. Chinese Herbal Medicines, 2020, 12(2): 104-117.

[4] G.E.S. Batiha, L.M. Alkazmi, L.G. Wasef, et al., Syzygium aromaticum L.(Myrtaceae): Traditional uses, bioactive chemical constituents, pharmacological and toxicological activities. Biomolecules, 2020, 10(2): 202.

[5] B. Shan, Y.Z. Cai, M. Sun, et al., Antioxidant capacity of 26 spice extracts and characterization of their phenolic constituents. Journal of Agricultural and Food
Chemistry, 2005, 53(20): 7749-7759.

[6] L.T. Hastuti, E. Saepudin, A.H. Cahyana, et al., The influence of sun drying process and prolonged storage on composition of essential oil from clove buds (Syzygium aromaticum). AIP Conference Proceedings, 2017, 1862(1): 030092.

[7] X. Han, T.L. Parker, and J. Dorsett, An essential oil blend significantly modulates immune responses and the cell cycle in human cell cultures. Cogent Biology, 2017, 3(1): 1340112.

[8] N.S. Mohammadi, H. Özgüneş, and N. Başaran, Pharmacological and toxicological properties of eugenol. Turkish Journal of Pharmaceutical Sciences, 2017, 14(2): 201.

[9] P.S. Kumar, R.M. Febriyanti, F.F. Sofyan, et al., Anticancer potential of Syzygium aromaticum L. in MCF-7 human breast cancer cell lines. Pharmacognosy Research, 2014, 6(4): 350.

[10] A.E. El-Hadary, H. Ramadan, and F. Mohamed, Hepatoprotective effect of cold-pressed Syzygium aromaticum oil against carbon tetrachloride (CCl4)induced hepatotoxicity in rats. Pharmaceutical Biology, 2016, 54(8): 1364-1372.

[11] N.A. Zahin, D. Tewari, M.T. Kabir, et al., Nanoparticles and its biomedical applications in health and diseases: Special focus on drug delivery. Environmental Science and Pollution Research, 2019: 1-18.

[12] H.H. Al-ahbabi, A.M. Jassim, and S.O. Hasson, Antimicrobial activity of aloe vera extract on cases of keratoconjunctivitisin sheep (in vivo and invitro study) and compared with penicillin-streptomycin. Basrah Journal of Veterinary Research, 2016, 15(2): 227-245.

[13] D. Chenthamara, S. Subramaniam, S.G. Ramakrishnan, et al., Therapeutic efficacy of nanoparticles and routes of administration. Biomaterials Research, 2019, 23(1): 1-29.

[14] A.M. Jasim, H.F. Hasan, and M.J. Awad, Preparation of vorapaxar loaded with vitamin E TPGS and PVA emulsified PLGA nanoparticles in vitro studies. Research Journal of Pharmacy and Technology, 2019, 12(9): 45034510.

[15] S. Asri-Rezaei, A. Nourian, A. Shalizar-Jalali, et al., Selenium supplementation in the form of selenium nanoparticles and selenite sodium improves mature male mice reproductive performances. Iranian Journal of Basic Medical Sciences, 2018, 21(6): 577.

[16] S.K. Sharma, D. Bolser, J. de Boer, et al., Construction of reference chromosome-scale pseudomolecules for potato: integrating the potato genome with genetic and physical maps. Genetics, 2013, 3(11): 2031-2047.

[17] A. Çelik, B. Mazmanci, Y.A. Çamlica, et al., Induction of micronuclei by lambda-cyhalothrin in Wistar rat bone marrow and gut epithelial cells. Mutagenesis, 2005, 20(2): 125-129.

[18] M.P. Hadidi, F,S. Haghani, and S.M. Jafari, Chitosan nanoparticles loaded with clove essential oil: Characterization, antioxidant and antibacterial activities. Carbohydrate Polymers, 2020: 116075.

[19] R.S. Hosseini, A. Rajaei, Potential Pickering emulsion stabilized with chitosan-stearic acid nanogels incorporating clove essential oil to produce fish-oilenriched mayonnaise. Carbohydrate Polymers, 2020, 241: 116340.

[20] P. Staňková, O. Kučera, H. Lotková, et al., The toxic effect of thioacetamide on rat liver in vitro. Toxicology in vitro, 2010, 24(8): 2097-2103.

[21] K. Das , Hepatoprotective and antioxidant activity of Lanneam coromandelica Linn. on thioacetamide induced hepatotoxicity in rats. International letters of Natural Sciences, 2014, 3.

[22] M.F. El-Maati, S.A. Mahgoub, S.M. Labib, et al., 
Phenolic extracts of clove (Syzygium aromaticum) with novel antioxidant and antibacterial activities. European Journal of Integrative Medicine, 2016, 8(4): 494-504.

[23] İ. Gülçin, M. Elmastaş, and H.Y. Aboul-Enein, Antioxidant activity of clove oil - A powerful antioxidant source. Arabian Journal of Chemistry, 2012, 5(4): 489499.

[24] K.G. Lee, T. Shibamoto, Antioxidant property of aroma extract isolated from clove buds [Syzygium aromaticum (L.) Merr. et Perry]. Food Chemistry, 2001, 74(4): 443448.

[25] K.M. Ahmed, E.M. Saleh, E.M. Sayed, et al., Antiinflammatory effect of different propolis extracts in thioacetamide-induced hepatotoxicity in male rat. Aust $J$ Basic App Sci, 2012, 6: 29.

[26] T.M. Chen, Y.M. Subeq, R.P. Lee, et al., Single dose intravenous thioacetamide administration as a model of acute liver damage in rats. International Journal of Experimental Pathology, 2008, 89(4): 223-231.

[27] T.G. Rodrigues, J.R. Fernandes, A. Sousa, et al., In vitro and in vivo effects of clove on pro-inflammatory cytokines production by macrophages. Natural Product Research, 2009, 23(4): 319-326.

[28] K.R. Park, J.H. Le, C. Choi, et al., Suppression of interleukin-2 gene expression by isoeugenol is mediated through down-regulation of NF-AT and NF- $\kappa \mathrm{B}$. International Immunopharmacology, 2007, 7(9): 12511258.

[29] H.M. Gashlan, A.B. Al-Beladi, Effects of clove oil on liver and antioxidant status of streptozotocin-induced diabetic rats. GARJMMS, 2017, 6: 103-110.

[30] P. Nasri, N. Hooshyar, B. Yalameha, et al., Ameliorative impact of hydroalcoholic extract of Clove buds (Syzygium aromaticum) on contrast-induced acute kidney injury: An experimental histopathological study. Journal of Nephropathology, 2019, 9(1): 1-4.

[31] D.E. Adli, K. Hachem, M. Benreguieg, et al., The efficiency of Syzygium aromaticum essential oil against renal intoxication by lead in rats during development. Bioscience Research, 2018, 15(3): 2126-2133.

[32] D. Abd Al-azem, H. Al-Derawi Karim, A. Sahar, et al., The protective effects of Syzygium aromaficum essential oil extract against methotrexate induced hepatic and renal toxicity in rats. Journal Pure Appl Microbiol, 2019, 13(1): 505-515.

[33] K.M. Al-Rawi, A. Khalafalla, Analysis of experimental agriculture Disgen.Dar Al-Kutub for printing and publishing. Mosul University, 2018, 6(3): 510.

[34] M. Hadidi, S. Pouramin, F. Adinepour, et al., Chitosan nanoparticles loaded with clove essential oil: Characterization, antioxidant and antibacterial activities. Carbohydrate Polymers, 2020, 236(15): 116075.

[35] C. Sebaaly, A. Jraij, H. Fessi, et al., Preparation and characterization of clove essential oil-loaded liposomes. Food Chemistry, 2015, 178: 52-62.

[36] G.E. Batiha, L.M. Alkazmi, L.G. Wasef, et al., Syzygium aromaticum L. (Myrtaceae): Traditional uses, bioactive chemical constituents, pharmacological and toxicological activities. Biomolecules, 2020, 10(2): 202.

[37] S. Hina, M. Shahid, and N. Jahan, In vitro antioxidant, hepatoprotective potential and chemical profiling of Syzygium aromaticum using HPLC and GC-MS. Pakistan Journal of Pharmaceutical Sciences, 2017, 30(3): 10311039.

[38] R.D. Coalson, A.M. Walsh, A. Duncan, et al., Statistical mechanics of a Coulomb gas with finite size particles: a lattice field theory approach. The Journal of Chemical Physics, 1995, 102(11): 4584-4594.

Copyright $\odot$ Adnan Mansour Jasim, Essa Daham Alhtheal, Saad Saleem Raheem, Kareem Judi Rawaa, and Abbas Hamad. This is an open-access article distributed under the terms of the Creative Commons Attribution License, which permits unrestricted use, distribution, and reproduction in any medium, provided the original author and source are credited. 